\title{
Medical education needs reform to improve student well-being and reduce burnout, say experts
}

n Cite as: CMAJ 2018 December 3;190:E1426-7. doi: 10.1503/cmaj.109-5685

Posted on cmajnews.com on Nov. 13, 2018.

$\mathrm{T}$ eaching and supporting wellness as a core competency of medicine may help reduce burnout in the profession, experts said at a recent conference on physician health.

Doctors suffer alarming rates of burnout, depression and suicide, and these problems often take root during training. More than a quarter of medical students internationally and a third of those in Canada report symptoms of burnout. Residency is a particularly vulnerable time; compared to Canadian physicians, medical residents face a $48 \%$ higher risk of burnout, $95 \%$ higher risk of depression and $72 \%$ higher risk of suicidal thoughts.

Research presented at the International Conference on Physician Health in Toronto indicated that the training environment, as well as personal factors, put some trainees at greater risk than others.

One longitudinal study of 4700 American trainees found that those who had higher anxiety and lower empathy in fourth year of medical school were more likely to burn out as residents, as were women and those who specialized in urology, neurology or emergency medicine. "Harassment and belittlement is likely to play a role," said lead author Dr. Lotte Dyrbye, who codirects the physician well-being program at the Mayo Clinic in Minnesota.

Another large cohort study of 2316 American medical students found that experiences of belittlement and harassment predicted higher levels of stress, worse physical and mental health, and suicidal thoughts. Students who weren't white and those who felt their schools

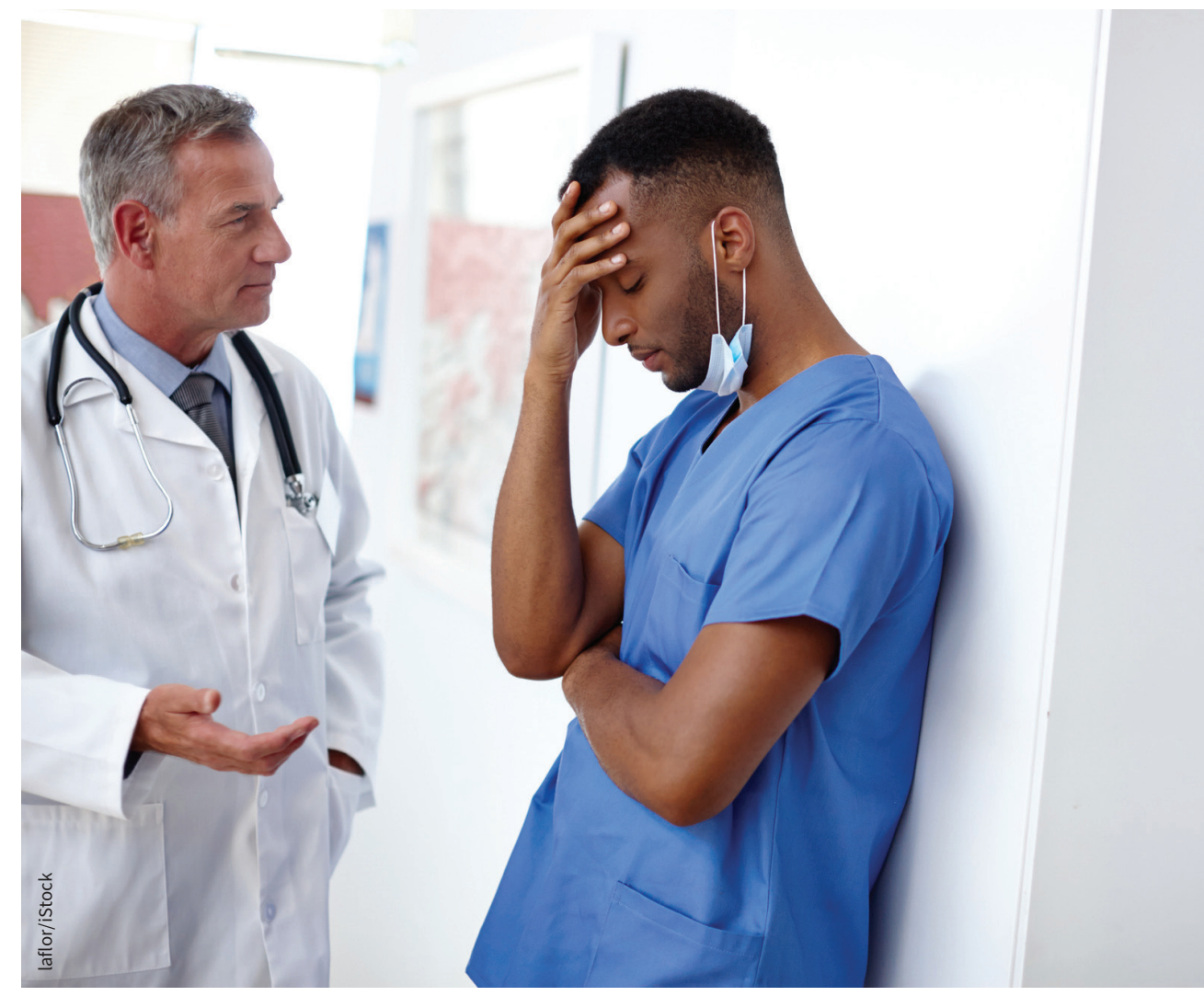

Medical trainees must learn that taking care of themselves is part of being a physician.

didn't try to minimize stress or encourage healthy extracurriculars were also more likely to report poor general health at graduation.

"There's a responsibility for our schools, for our training programs, to have a positive work environment and learning environment, to make sure faculty are wellequipped, and to pace the curriculum in the right way," said Dyrbye.

Although most medical schools provide some wellness training, it tends to be evalu- ated on student participation, "not if it made a difference," she said. Even among schools involved in a national initiative to modernize medical education, "only $22 \%$ had any kind of competency" or formal requirement about the skills students must demonstrate related to well-being.

Making wellness a core competency of medicine would unlock resources to "design, implement and evaluate curriculum aimed at helping students do their part and perhaps send a message that taking 
care of yourself is a central part of being a physician," said Dyrbye. It may also spur schools to "work on the learning environment and engage in faculty development."

According to Dr. Kimberly Lomis, vice president of undergraduate medical education innovations at the American Medical Association, the traditional model of two years of lectures followed by two years of clinical rotations doesn't prepare students for the realities of practice. "Having material delivered to you that you regurgitate back is very different from what you have to do throughout your career, and this is part of the stress of being a physician downstream."

Medical schools are beginning to provide more hands-on experience earlier in training to reduce the shock of transition to clerkship and residency. "That is going to foster stronger professional identity and a greater sense of meaning right from the start," Lomis said.

Classrooms are also shifting away from lectures and rote memorization to emphasize problem solving in teams, an area where many trainees struggle. "Many of our students have wrapped up their identity in the fact that they know things, so to sit in a group and acknowledge that they don't is important for their wellbeing in the long term."

Teaching students more about how to navigate the health system and advocate for change may also combat feelings of powerlessness that contribute to burnout, Lomis said. "Many physicians feel like they're victims of the system and partially that's because they don't understand how it works or how to influence it."

Major reforms can pose major pitfalls, however, Lomis noted. For example, taking away lectures can be "unsettling" for students who have built their academic success on that format. Likewise, adopting a more open-ended approach to evaluating students in which there are "multiple pathways" to demonstrate competency may combat perfectionism in some, while others "say it just feels like there are more ways to fail."

Schools also need to counter the common perception of wellness training as "busy work," Lomis said. "Wellness is not easy; it's actually hard and requires a lot of thought and skill and ongoing work throughout your career, and I think if we frame it that way, and build a safe environment, then these structural changes can improve their outcomes downstream."

Lauren Vogel, CMAJ 